\title{
Dielectric behavior and electrical conductivity of PVDF filled with functionalized single-walled carbon nanotubes
}

\author{
J.A. Puértolas ${ }^{1 *}$, J.F. García-García ${ }^{1}$, F.J. Pascual ${ }^{1}$, J.M. González-Domínguez² ${ }^{2}$ M.T. \\ Martínez $^{2}$ A. Ansón-Casaos ${ }^{2}$ \\ ${ }^{1}$ Department of Materials Science and Technology, Instituto de Investigación en Ingeniería \\ de Aragón, I3A, Universidad de Zaragoza, 50018 Zaragoza, Spain \\ ${ }^{2}$ Instituto de Carboquímica ICB-CSIC, Miguel Luesma Castán 4, 50018 Zaragoza, Spain
}

*Correspondence to Prof. José A. Puértolas

Department of Materials Science and Technology,

Escuela de Ingeniería y Arquitectura-I3A, Universidad de Zaragoza,

E-50018, Zaragoza, Spain

Tel. : +34 976762521

Fax. : +34 976761957

e-mail: japr@unizar.es 


\begin{abstract}
Polyvinylidene fluoride (PVDF)/ single-walled carbon nanotube (SWCNT) composites are characterized by X-ray diffraction and scanning calorimetry, and studied by dielectric relaxation spectroscopy (DRS) in the temperature range of -75 to $150^{\circ} \mathrm{C}$. The effects of SWCNTs and SWCNT functionalization are analyzed in terms of $\alpha$ and $\alpha_{c}$ relaxation, dielectric permittivity, loss tangent, and AC electrical conductivity. Some small changes are detected in $\alpha$ relaxation with the addition of SWCNTs, and a strong influence of SWCNTs is observed in the other relaxation $\alpha_{\mathrm{c}}$. Below the percolation threshold, the dielectric permittivity of functionalized SWCNT composites increases compared to blank PVDF, without notable changes in the dielectric loss. All the composite systems show an electrical percolation behavior with different thresholds depending on SWCNT functionalization. Threshold concentrations remain nearly unchanged in the whole temperature and frequency ranges. The base PVDF conductivity strongly depends on temperature and frequency, while the maximum conductivity above the percolation remains nearly unchanged $\left(\sim 10^{-2} \mathrm{~S} / \mathrm{m}\right)$ for all the systems, temperatures and frequencies.
\end{abstract}

KEYWORDS: Polyvinylidene fluoride, carbon nanotube, polymer relaxations, permittivity, alternating current, temperature 


\section{INTRODUCTION}

Polyvinylidene difluoride (PVDF) is a thermoplastic polymer with a semicrystalline microstructure and a varied polymorphism, showing high compliance, thermal and chemical stability, and piezoelectric and pyroelectric properties. These properties are the base of different applications such as tactile sensors, infrared imaging devices, actuators, electrolyte-gated transistors, etc. [1,2]. The incorporation of different nanofillers is a way to further improve the performance of raw PVDF and extend its applications. Probably, carbon nanotubes (CNTs) are among the most studied reinforcements due to their intrinsic characteristics including high aspect ratio, outstanding electrical and mechanical properties, and versatility for surface functionalization. The CNT/PVDF composites enable new applications in different sectors, including membranes for ultrafiltration [3], superhydrophobic surfaces [4] fuel cell electrodes, charge transport layers in sensor systems [5], high energy storage systems [6, 7], infrared detectors [8], electromagnetic interference shielding and radar absorbing coatings for military energy systems [9], electrodes for electrocardiograms [10], actuators and pressure sensors [11, 12], in-vitro muscle cell growth [13], and structural health monitoring systems [14]. A comprehensive description of the applications of CNT-based PVDF composites can be found in several reviews $[15,16]$.

The incorporation of small amounts of CNTs in an insulating polymer leads to an increase in the electrical conductivity by several orders of magnitude due to the percolation effect. The conducting network formation strongly depends on the onedimensional character of CNTs and their suitable dispersion in the polymer matrix. The increase in conductivity is also associated to a strong variation in the dielectric constant, which is due to the insulating-conductor transition around the CNT percolation threshold. Such an increase in the dielectric constant is positive in some applications, for instance 
energy storage in capacitors. However, it is usually accompanied by a strong increase in the loss tangent that reduces the composite potential applications. Therefore, it is necessary to find a way for increasing the PVDF dielectric constant, while maintaining the composite dielectric losses down at a suitable level. Different strategies have been developed in order to resolve this paradigm, including the incorporation of a secondary $\mathrm{BaTiO}_{3}$ insulating filler [17], the surface oxidation of metallic nanowires [18], and others that have been widely summarized by Arjmand et al. [19]. One of the routes is based on surface modification of CNTs with different functionalization types including surface oxidation [20], nitrogen doping [21], and the incorporation of organic compounds such as polypyrrole [6]. These functionalizations have been mostly performed on multi-walled carbon nanotubes (MWCNTs), although single-walled carbon nanotubes (SWCNTs) have a higher aspect ratio and thus they are expected to make possible lower percolation thresholds and a larger density of nanocapacitors than MWCNTs. Similarly to MWCNTs, surface chemical modification of SWCNTs may be a way towards PVDF composites with high dielectric permittivity and low loss. So far, SWCNTs have been chemically functionalized with the aim of increasing the PVDF electrical conductivity and decrease the percolation threshold [22-25]. However, new studies are still needed to understand the effects of chemical functionalization on the macroscopic dielectric and electrical properties.

In this work we analyze the dielectric and conductive behavior of SWCNT/PVDF composites, where SWCNTs are untreated, functionalized by nitric acid treatment or functionalized with fluoroalkyl moieties. Previously, we have reported the mechanical elastic properties of the materials [24]. Here, the effect of nanofiller chemical modifications on PVDF dielectric relaxation and alternating current (AC) conductivity is studied in the temperature range of -75 to $150^{\circ} \mathrm{C}$. Plots of the electrical conductivity vs. 
SWCNT filler concentration are presented as a function of both temperature and frequency, allowing a study of the percolation threshold in the whole measured temperature and frequency ranges.

\section{MATERIALS AND METHODS}

For the current study, SWCNTs (AP-SWNT grade) were purchased from Carbon Solutions Inc., Riverside, CA, United States. This SWCNT powder material is synthesized by the electric-arc reactor method using Ni/Y catalysts and contains $<30$ wt.\% metal residue. PVDF powder $\left(\mathrm{M}_{\mathrm{w}} \sim 534,000\right.$, ref. 182702) was purchased from Sigma-Aldrich, Madrid, Spain.

Pristine SWCNT powders were utilized as-received or functionalized by two different chemical procedures: nitric acid treatment (SWCNT-NIT) and functionalization with perfluorooctyl moieties (SWCNT-PFO). The functionalization procedures and the SWCNT/PVDF composite preparation have been previously described [24]. The SWCNTNIT material was prepared by treatment with $1.5 \mathrm{M} \mathrm{HNO}_{3}$ in a reflux for $2 \mathrm{~h}$. Oxygen functional groups are created during the nitric acid treatment, and most of the metal catalyst residues are eliminated. The PFO-SWCNT material was synthesized through the covalent bonding of heptadecafluorooctyl phenyl groups $\left(\mathrm{F}_{3} \mathrm{C}-\left(\mathrm{CF}_{2}\right)_{7}-\mathrm{C}_{6} \mathrm{H}_{4^{-}}\right)$to pristine SWCNTs, following the diazonium salts route. A complete characterization of the SWCNT functionalization is included in the Supplementary Data.

The preparation of SWCNT/PVDF composites consisted of a premixing stage in acetone followed by hot pressing inside a stainless steel round mold $(\varnothing=13 \mathrm{~cm})$. A total mass of $11 \mathrm{~g}$ of SWCNTs + PVDF was suspended in $\sim 100 \mathrm{~mL}$ of acetone with the help of an ultrasound bath. The mixture was kept in the ultrasound bath and at once stirred with a blade mixer until acetone was evaporated. Then the material was dried in an oven to 
remove the trapped solvent. Finally, it was hot-pressed at $200^{\circ} \mathrm{C}$ under $2 \mathrm{Tm}$ for $2 \mathrm{~h}$ in a Specac laboratory press and left to cool down naturally inside the mold without any further pressure. Hereafter, the composites will be referred to as SWCNT-X/PVDF, SWCNT-NIT-X/PVDF, and SWCNT-PFO-X/PVDF for the non-functionalized, acidtreated and fluoroalkyl-functionalized SWCNTs, respectively, where X stands for the filler weight percentage, according to Table 1.

\section{Structural characterization and dielectric measurements}

X-ray diffraction (XRD) was performed in a Bruker AXS D8 Advance diffractometer using $\mathrm{CuK} \alpha$ radiation. The polymer crystallinity $\left(\mathrm{X}_{\mathrm{XRD}}\right)$ was calculated from data in the range of $2 \theta=12-24^{\circ}$. Differential scanning calorimetry (DSC) experiments were performed in a Mettler TA4000 calorimeter. The polymer crystallinity $\left(\mathrm{X}_{\mathrm{DSC}}\right)$ was calculated as: $\mathrm{X}_{\mathrm{DSC}}=\Delta_{\mathrm{m}} \mathrm{H} / \Delta_{\mathrm{m}} \mathrm{H}_{100}$, where $\Delta_{\mathrm{m}} \mathrm{H}_{100}$ represents the melting enthalpy of a $100 \%$ crystalline sample of pure PVDF, which is $104.6 \mathrm{~J} \mathrm{~g}^{-1}$ [26]. Some additional details of the XRD and DSC analysis are given in the Supplementary Data.

Dielectric measurements were performed using a dielectric spectrometry setup (Novocontrol BDS4000), which includes a two-terminal dielectric cell, a frequency response analyzer (Solartron 1250), and a high impedance preamplifier of variable gain. The complex dielectric constant was obtained by sweeping in the frequency range of 0.1 $\mathrm{Hz}$ to $10^{5} \mathrm{~Hz}$ at different stabilized temperatures by heating from $-75{ }^{\circ} \mathrm{C}$ to $150{ }^{\circ} \mathrm{C}$ in temperature steps of $5{ }^{\circ} \mathrm{C}$. The samples were disks of a $12 \mathrm{~mm}$ of thickness, which were held between the condenser plates. The dielectric analysis was conducted through the complex dielectric permeability $\varepsilon^{*}=\varepsilon^{\prime}-\varepsilon^{\prime \prime}$, taking into account the dielectric constant $\varepsilon^{\prime}$ and the dielectric loss $\varepsilon^{\prime \prime}$, as well as the $\tan \delta=\varepsilon^{\prime \prime} / \varepsilon^{\prime}$. The dielectric relaxations were analyzed in the isotherm spectra by fitting the response in the frequency domain to Havriliak-Negami (HN) curves (Eq. 1) [27, 28] 


$$
\varepsilon^{*}-\varepsilon_{\infty}=\frac{\Delta \varepsilon}{\left\{1+\left(i w \tau_{H N}\right)^{\alpha}\right\}^{\beta}} \quad(E q .1)
$$

where $\omega=2 \pi f$ is the frequency in radians/second, $\alpha$ and $\beta$ are shape parameters respectively corresponding to the width and asymmetry of the relaxation time distributions, $\tau_{H N}$ is the Havriliak-Negami relaxation time, and $\Delta \varepsilon=\varepsilon_{S}-\varepsilon_{\infty}$ is the relaxation strength $\left(\varepsilon_{S}\right.$ and $\varepsilon_{\infty}$ are the real part of the permittivity when $\omega \rightarrow 0$ and $\omega \rightarrow \infty$, respectively).

At sufficiently high temperatures, ohmic conduction due to charge carriers $\sigma^{*}$ frequently dominates the loss contribution at low frequencies, potentially masking dielectric relaxations. In order to discriminate dielectric and conductivity effects, the conduction-free dielectric loss $\varepsilon^{\prime \prime}{ }_{N C}$ was also determined by Eq. 2 [29]

$$
\varepsilon^{\prime \prime}{ }_{N C}=\varepsilon^{\prime \prime}-\frac{\sigma_{0}}{\varepsilon_{0} 2 \pi f^{S}}(E q .2)
$$

with $f$ being the frequency in Hertz, $\sigma_{0}$ a pre-exponential coefficient, and $s$ an exponent normally close to 1 .

\section{RESULTS AND DISCUSSION}

\section{Structure and microstructure}

Five crystalline forms of PVDF have been identified, the most common ones being the $\alpha$ and $\beta$ phases. The $\alpha$-PVDF is easily produced from the polymer melt, while the $\beta$ PVDF, which is piezoelectric and pyroelectric, can be synthesized by crystallization from a solution or by drawing $\alpha$-PVDF. Figure 1 shows XRD and DSC results for neat PVDF and three SWCNT/PVDF composites with filler contents above the electrical percolation threshold. XRD analysis indicates that crystalline polymer domains are $\alpha$-phase in all the cases. A decrease in the polymer crystallinity is calculated with increasing SWCNT 
contents (Table 2). This fact is in part associated to a certain decrease in the (020) band intensity, which is most clearly observed for the SWCNT-NIT-7/PVDF material.

The presence of SWCNTs causes an increase in the PVDF crystallization temperature, and a decrease in the crystallization enthalpy (Table 2). The increase in the PVDF crystallization temperature upon the filler addition indicates that SWCNTs act as nucleation agents during crystallization. On the contrary, melting temperature does not substantially change upon the addition of SWCNTs. Melting enthalpy decreases with increasing SWCNT contents, indicating a decrease in the PVDF crystallinity, in agreement with XRD results. The melting temperature $T_{m}$ is commonly applied to estimate the lamellar thickness $\mathrm{L}_{\mathrm{c}}$ through the Gibbs-Thomson equation:

$$
T_{m}=T_{m, 0}\left(1-\frac{2 \sigma}{L_{c} \rho_{c} \Delta H_{m}^{0}}\right)(E q .3)
$$

where $T_{m}$ is the polymer melting temperature, $T_{m, 0}$ the equilibrium melting temperature of a perfect crystalline polymer, $\sigma$ the specific surface energy, $\rho_{c}$ the density of the crystalline phase, and $\Delta \mathrm{H}_{\mathrm{m}}^{0}$ the melting enthalpy of a perfect crystalline polymer. Since melting temperatures are nearly identical for PVDF and all the composites, it is deduced that the thickness of PVDF crystalline lamellae does not substantially change. Therefore the decrease in the crystallinity degree might be associated to a reduction in the lateral size of crystalline lamellae. The presence of nanotubes initially favors polymer chain associations, while it decreases the long range order inside the lamellae. These microstructural effects, associated to the thermal response, have been previously observed in PVDF composites with double-walled CNTs [30], non-functionalized MWCNTs [31], other carbon-based nanoadditives [25], and even in functionalized MWCNT/PVDFPMMA blends [32].

Another outcome of the X-ray diffractograms is that SWCNTs did not induce the formation of $\beta$-phase PVDF in our composites. This fact was also confirmed by infrared 
spectroscopy (Supplementary Data). The observed behavior is different from that in some recent studies, as the presence of the reinforcement introduced dramatic changes in the $\beta$ phase percentage [33-36]. Moreover, Sharma et al. [36] reported that $\beta$-phase formation leads to the disappearance of certain dielectric relaxations. In our case, SWCNTs contribute to an increase in the $\alpha_{c}$ relaxation strength, as we will further comment in the next section, indirectly confirming that $\beta$-phase PVDF is not formed.

\section{Dielectric relaxations}

Figure 2 illustrates 3D temperature/frequency representations of the $\varepsilon^{\prime}$ and $\varepsilon$ '” response corresponding to neat PVDF. Analogous maps were carried out for all the studied composites. For the unfilled material, both maps clearly show the presence of two relaxations at low/intermediate temperatures and a strong conductivity contribution in the highest temperature range. The relaxations will be labelled as $\alpha$ and $\alpha_{c}$ following most of the dielectric and mechanical measurements that can be found in the literature concerning the non-polar $\alpha$-PVDF phase. The $\alpha$ relaxation is associated to the dynamic glass transition $\mathrm{T}_{\mathrm{g}}$, and thus it is related to cooperative segment motions within the main chain of the amorphous polymer region. The $\alpha_{c}$ relaxation is related to different imperfections of the $\alpha$-phase crystallites, such as chain loops at the lamellae surface, discontinuities, etc. At temperatures below $-70{ }^{\circ} \mathrm{C}$, the material also presents the $\beta$ relaxation, which is a secondary transition related to local movements of polar groups in polymeric chains. Our measurements did not allow the observation of $\beta$ relaxation, since our initial temperature was not low enough.

\section{$\alpha$ relaxation}

The isochronous curves $\varepsilon^{\prime}(\mathrm{T})$ and $\varepsilon^{\prime \prime}(\mathrm{T})$ are plotted in Figure 3 for two frequencies, $10 \mathrm{~Hz}$ and $10^{5} \mathrm{~Hz}$, as an example. In blank PVDF, the first relaxation $\alpha$ 
appears in the low temperature range, from $-40{ }^{\circ} \mathrm{C}$ to $-10{ }^{\circ} \mathrm{C}$ at $10 \mathrm{~Hz}$, and it is observed as a step in $\varepsilon^{\prime}(\mathrm{T})$ and as a peak centered around $-30{ }^{\circ} \mathrm{C}$ in $\varepsilon^{\prime \prime}(\mathrm{T})$ at $10 \mathrm{~Hz}$, being at higher temperatures for $10^{5} \mathrm{~Hz}$. In order to show the influence of CNT functionalization on the dielectric relaxation, we have chosen filler concentrations below the percolation threshold, when the composites show a reduced contribution of the conductivity term. Percolation thresholds are approximately 1,5 and $3 \mathrm{wt} \%$ respectively for SWCNT/PVDF, SWCNTNIT/PVDF and SWCNT-PFO/PVDF composites (see the "percolation threshold" section). Inside the concentration range below the threshold, we have chosen the maximum measured concentration to observe the effects in the best possible way. Therefore, Figure 3 depicts the isochronal curves for SWCNT-1/PVDF, SWCNT-NIT-5/PVDF and SWCNT-PFO-3/PVDF composites. In these composites, the temperature region in which the $\alpha$ relaxation appears is nearly identical and the band maximum in $\varepsilon^{\prime \prime}(\mathrm{T})$ curves is shifted by less than 3 degrees, so changes in $T_{g}$ provoked by SWCNT fillers should be small, in agreement with previous results [11]. In composites with filler concentrations above the percolation threshold, AC conductivity is predominant, and it will be discussed later.

In order to further analyze the $\alpha$ relaxation, isothermal curves $\varepsilon^{\prime \prime}(\mathrm{f})$ are plotted in Figure 4 and fitted to an $\mathrm{HN}$ contribution (Eq. 1) for the dielectric term plus a conductivity contribution with a frequency dependence (Eq. 2). For blank PVDF, the following characteristics are observed as the temperature increases: i) an increase in the relaxation strength, ii) a peak sharpening and iii) an increase in the asymmetry, with the respective Eq. 1 parameters ranging from $\Delta \varepsilon=3.7, \alpha=0.24, \beta=1$ at $-40{ }^{\circ} \mathrm{C}$ to $\Delta \varepsilon=4.7, \alpha$ $=0.64, \beta=0.39$ at $-10{ }^{\circ} \mathrm{C}$ (Figure 4a). The behavior is similar to that observed by Sencadas et al. [37], except for the relaxation strength, which remained nearly constant at $\Delta \varepsilon=5.2$ in their study. The HN fit also provides information about the relaxation time 
$\tau_{\mathrm{NH}}$. Figure 5a shows the temperature evolution of $\tau_{\mathrm{NH}}$ in an Arrhenius-type plot, which allows the calculation of an apparent activation energy $E_{a p p}=1.11 \mathrm{eV}$ for unfilled PVDF. Such a value is similar to others obtained by the dielectric relaxation spectroscopy (DRS) technique: $\mathrm{E}_{\mathrm{app}}=1.08 \mathrm{eV}$ [38], $1.05 \mathrm{eV}$ [39]. However, the narrow temperature range of the $\alpha$ relaxation does not allow us to observe the real non-Arrhenius behavior associated to the glass transition. As it was clearly described by Kaszynska et al. [40] using nuclear magnetic resonance (NMR), dielectric and mechanical data, the complete temperature dependence of $\tau_{\mathrm{NH}}$ can be well fitted to a Vogel-Tamman-Fulcher law. In Sencadas [37], the non-Arrhenius behavior was also apparent just by DRS, as the temperature range valid for $\tau_{\mathrm{NH}}$ calculation resulted to be wider than in our measurements.

In order to study the influence of carbon nanotubes on $\alpha$ relaxation from $\varepsilon^{\prime \prime}(\mathrm{f})$ plots, it is necessary to separate the conductivity from dielectric contributions. This separation can be only performed if AC conductivity is not predominant, i.e. in all the composites with SWCNT concentrations above the percolation threshold. Figures $\mathbf{4 b}$ and $4 \mathbf{c}$ show the evolution of $\varepsilon^{\prime \prime}(\mathrm{f})$ spectra after subtracting the conductivity term in SWCNTNIT-5/PVDF and SWCNT-PFO-3/PVDF respectively. The results point out that the incorporation of carbon nanotubes into PVDF introduces some small changes in the $\alpha$ relaxation. Firstly, the relaxation strength increases, reaching a maximum value of $\Delta \varepsilon=$ 1.6 at $-10{ }^{\circ} \mathrm{C}$, whereas $\Delta \varepsilon=0.9$ in the neat PVDF. Analogous changes occur for the rest of temperatures at which $\alpha$ is present. This behavior is in the line of the increments arisen in the $\alpha$ relaxation region of the $\varepsilon^{\prime}(\mathrm{T})$ plot (Figure 3). The increase in $\Delta \varepsilon$ could be associated to the decrease in crystallinity, which is close to $10 \%$ (Table 2) and is given by an increase in the amount of the free amorphous chains involved in $\alpha$ relaxation. Another effect of SWCNT fillers is a small shift in the frequency of the $\varepsilon^{\prime \prime}$ maximum, which implies that the relaxation time $\tau_{\mathrm{NH}}$ becomes shorter in composites. Figure 5a reflects the 
Arrhenius behavior of $\tau_{\mathrm{NH}}$ with activation energies around $1.0 \mathrm{eV}$ in SWCNT-NIT/PVDF and $0.90 \mathrm{eV}$ in SWCNT-PFO/PVDF. These results would indicate a higher molecular mobility in the amorphous domain and therefore a reduction in $\mathrm{T}_{\mathrm{g}}$, as it was confirmed by Anson-Casaos et al. by dynamical mechanical analysis (DMA) [24].

$\alpha_{c}$ relaxation

The other relaxation $\alpha_{c}$ appears in the $10-80{ }^{\circ} \mathrm{C}$ range both in the neat PVDF and in the composites. This relaxation feature was described for the first time by Kakutani et al. [41] and later by $[24,42,43]$ using DMA. However, the $\alpha_{c}$ relaxation has seldom been analyzed in detail by DRS: the relaxation does appears neither in Sencadas et al. [37] nor in Yuan et al. [44] works, whereas in other studies its presence is only detected at the lowest frequencies or it is overlapped with the $\alpha$ relaxation $[39,42,45,46]$, and therefore it does not allow to obtain reliable parameter fittings.

The two-contribution model considering $\mathrm{HN}$ relaxation and $\mathrm{AC}$ conductivity remains valid. The outcomes for virgin PVDF show that the relaxation strength of $\alpha_{c}$ is nearly constant with a leger increase from $\Delta \varepsilon=4$ at $15^{\circ} \mathrm{C}$ to $\Delta \varepsilon=5$ at $80^{\circ} \mathrm{C}$. According to Mijovic et al. [47], the trend does not continue above $80{ }^{\circ} \mathrm{C}$, and the peak magnitude undergoes a decrease with temperature up to its disappearance at around $177^{\circ} \mathrm{C}$, caused by the crystalline origin of the $\alpha_{c}$ relaxation. The fitting process in our data gave shape parameters around $\alpha=0.5$ and $\beta=1$ for all the isothermal spectra. The Arrhenius plot of $\tau_{\mathrm{NH}}$ gave a straight line with $\mathrm{E}_{\mathrm{a}}=0.92 \mathrm{eV}$, which is lower than $1.2 \mathrm{eV}$ obtained in [39] and similar to $0.95 \mathrm{eV}$ obtained by Mijovic et al. [47] and $0.93 \mathrm{eV}$ by Linares et al. [48], thus confirming the Arrhenius character of the $\alpha_{c}$ relaxation for neat PVDF.

CNTs induce a strong increase in the $\alpha_{c}$ dielectric strength in the studied temperature range, from $\Delta \varepsilon=1$ at $15^{\circ} \mathrm{C}$ to $\Delta \varepsilon=7$ at $80^{\circ} \mathrm{C}$ in SWCNT-NIT5/PVDF, and from $\Delta \varepsilon=4$ to $\Delta \varepsilon=10$ in SWCNT-PFO-3/PVDF. These changes are accompanied by 
increases in symmetry and broadness. Figure $\mathbf{5 b}$ shows the temperature dependence of $\tau_{\mathrm{NH}}$ in an Arrhenius plot, whose points do not follow strictly a straight line. Data fitting to an Arrhenius behavior gave $\mathrm{E}_{\mathrm{a}}=0.8-0.9 \mathrm{eV}$ for the composites, just a little lower than the values obtained for $\alpha$ relaxation. However, the SWCNT presence notably increases the $\alpha_{c}$ relaxation times of PVDF, in agreement with observations for PVDF/PMMA blends after the addition of 1 wt.\% of MWCNTs [36]. The effect can be associated to the interaction between the fillers and the matrix, which affects the mobility of the chains.

The mechanism involved in $\alpha_{c}$ relaxation, according to NMR data by McBrierty et al. [49], is associated to motions of folded chains on the surface of crystal lamellae, which are more restricted than those undergone by amorphous chains. The relaxation times obtained in $\alpha_{c}$ relaxation, which are two orders of magnitude slower than in $\alpha$ relaxation, support that explanation. From DMA measurements, Alves et al. [43] found some effects of thermal treatments on $\alpha_{c}$ and $\alpha$ relaxations. The conclusion was that $\alpha_{c}$ relaxation is related to the annealing process suffered by the samples. In order to deepen in this aspect, we carried out a thermal cyclic process which consisted of one heating process from $-45^{\circ}$ $\mathrm{C}$ to $185^{\circ} \mathrm{C}$ followed by a cooling process at $10{ }^{\circ} \mathrm{C} / \mathrm{min}$ up to $-45^{\circ} \mathrm{C}$ and a second heating process. The results of $\alpha_{c}$ relaxation before and after the second melting process were quite similar, with only a $15 \%$ variation in the relaxation strength. Such a small change could be related to changes in the specimen shape inside the measurement space or to changes in the degree of crystallinity.

\section{Dielectric constant and loss tangent}

The presence of CNTs significantly affects the PVDF dielectric constant $\left(\varepsilon^{\prime}\right)$. Table 3 shows the $\varepsilon^{\prime}$ values together with the loss tangent $(\tan \delta)$ at two frequencies $\left(1 \mathrm{~Hz}\right.$ and $10^{5}$ 
$\mathrm{Hz})$ and two temperatures $\left(25\right.$ and $\left.150^{\circ} \mathrm{C}\right)$ for two materials of each composite group with concentrations below and above their respective percolation thresholds.

At a low frequency $(1 \mathrm{~Hz})$, the dielectric constant changes from $\varepsilon^{\prime}=12$ for PVDF to $\varepsilon^{\prime}=124$ for SWCNT-1/PVDF. The rise in the dielectric constant has its origin in the presence of conductive nanofillers, which contribute to the formation of a randomly distributed network of nanocapacitors, increasing the material polarization via the Maxwell-Wagner-Sillars mechanism [50]. When the filler concentration is near the percolation threshold, nomadic charge carriers are blocked at the interfaces between CNTs and the matrix [50]. In our measurements, the effect is much less pronounced for the composites with functionalized SWCNTs, even at higher filler concentrations. Dielectric constants reach $\varepsilon^{\prime}=22$ and $\varepsilon^{\prime}=14$ respectively for SWCNT-PFO-3/PVDF and SWCNTNIT-5/PVDF, which still have filler concentrations well below their respective percolation thresholds. The difference between both composites might be associated to the different dipolar contribution of the functional groups. At the highest frequency $\left(10^{5} \mathrm{~Hz}\right)$, the influence of SWCNTs in the dielectric constant is lower than at $1 \mathrm{~Hz}$. Values change from $\varepsilon^{\prime}=9.4$ for PVDF to $\varepsilon^{\prime}=21$ for SWCNT-1/PVDF and $\varepsilon^{\prime}$ around 15 for the composites with functionalized CNTs. Whilst interfacial polarization is predominant at low frequencies, the mechanism of PVDF dipolar polarization could prevail at the moderately high frequency regime around $10^{5} \mathrm{~Hz}$.

Table 3 also reflects the strong increase in the dielectric constant of the composites when the CNT concentration just exceeds the percolation, reaching at $10^{5} \mathrm{~Hz}$ values of $\varepsilon^{\prime}$ $=105,50$ and 43 for SWCNT-2/PVDF, SWCNT-PFO-4,6/PVDF and SWCNT-NIT7/PVDF, respectively. In any case, the values are still very far from the $\varepsilon^{\prime}=600$ of SWCNT-3/PVDF, which is a composite with a concentration clearly above the percolation. These strong increases are in agreement with the critical behavior of the 
dielectric constant near a percolation threshold of a heterogeneous system, in particular in CNT/PVDF composites $[51,52]$. The concentration dependence typically follows the law $\varepsilon^{\prime} \sim\left(\Phi_{c}-\Phi\right)^{-q}$, where $\Phi_{c}$ is the critical concentration at the percolation threshold, $\Phi$ the concentration of CNTs and q a scaling constant [53]. In the present work, the limited number of concentrations in the sample series does not allow to calculate the exact value of the percolation critical concentration $\Phi_{c}$.

Another characteristic of the dielectric constant is the presence of the "wave" effect, which is defined as the appearance of a maximum in the isochronous curve $\varepsilon^{\prime}(\mathrm{T})$. This phenomenon was observed by Li et al. [54] in the neat PVDF matrix and also in MWCNT/PVDF composites. In our work, the effect is only observed in SWCNT-X/PVDF composites at temperatures around $100{ }^{\circ} \mathrm{C}$ (see SWCNT-1/PVDF curves in Figure 3a). Following $\mathrm{Li}$ et al. [54], the decrease in $\varepsilon^{\prime}$ at around $100^{\circ} \mathrm{C}$ occurs when the semicrystalline polymer changes into a rubbery state, making easier for the fillers to connect with each other. A decrease in the density of individual interfaces between the nanofiller and the matrix is induced and consequently a decrease in the interfacial polarization occurs. In a certain way the effect is reminiscent of the decrease of $\varepsilon^{\prime}$ when CNTs concentration surpasses $\Phi_{c}$. In our data, the wave effect is hindered by CNT functionalization, possibly due to the different separation, in terms of filler wt.\%, of the chosen specimens from their respective percolation threshold.

Regarding the loss tangent, Table 3 reflects that SWCNTs cause a strong increase at concentrations above the percolation threshold. The addition of untreated SWCNTs, even at relatively low amounts, leads to an increase in $\tan \delta$, from 0.07 for neat PVDF to 1.16 for SWCNT-1/PVDF at $1 \mathrm{~Hz}$ and $25^{\circ} \mathrm{C}$, while higher amounts of functionalized SWCNTs can be added with only small changes in tan $\delta$. A qualitative difference exits between the untreated and functionalized SWCNT composites, since $\tan \delta$ values for 
functionalized SWCNT composites are systematically lower when they are compared under identical conditions (above or below the percolation threshold, constant temperature and frequency). For composites with functionalized SWCNTs below the percolation threshold (SWCNT-PFO-3/PVDF and SWCNT.NIT-5/PVDF) the loss tangent remains near that of blank PVDF, while certain increments in the dielectric constant are registered at once. The effect might be related to the role of functional groups as protective insulating layers, which hinder charge losses through the SWCNT network. Moreover, the fluoroalkyl moiety shows a somewhat greater effect than the oxygen functional groups inserted by the nitric acid treatment.

\section{Electrical conductivity}

When the electrical conductivity $(\sigma)$ becomes relevant in insulating materials, the analysis of experimental data can be done through the $\log \sigma$ vs. $\log \omega$ representation. This plot provides a horizontal straight line for the frequencies in which the direct current (DC) conductivity is predominant and a dispersion region above a critical frequency $\omega_{c}$, where the conductivity increases with frequency according to equation 3:

$$
\sigma(\omega)=\sigma_{\mathrm{dc}}+\sigma_{\omega} \omega^{\mathrm{s}} \quad(E q 3)
$$

where $\sigma_{\mathrm{dc}}$ is the frequency-independent DC conductivity, $\sigma_{\omega}$ is a constant and $\mathrm{s}$ a parameter that takes values of $0<s<1$. Figure 6 shows the $\log \sigma$ vs. $\log \omega$ for the PVDF matrix and the SWCNT/PVDF composites at room temperature and $150{ }^{\circ} \mathrm{C}$. Continuous lines in the figures represent fittings to Equation 3. The outcomes reflect that conductivity increases with temperature and most especially with CNT addition.

DC conductivity analysis 
Concerning the influence of temperature in the neat PVDF resin, an increase of three orders of magnitude in DC conductivity, from $10^{-11} \mathrm{~S} / \mathrm{m}$ to $10^{-8} \mathrm{~S} / \mathrm{m}$, occurs when the temperature increases from $25^{\circ} \mathrm{C}$ to $150{ }^{\circ} \mathrm{C}$. In the range of $125-150{ }^{\circ} \mathrm{C}$, where the presence of relaxations does not predominate in $\varepsilon^{\prime \prime}$ and consequently $\sigma(\omega)$ follows the behavior given by Equation 3, the as calculated $\sigma_{\mathrm{dc}}$ follows an Arrhenius behavior (Figure 7), with an activation energy close to $E_{a}=1.66 \mathrm{eV}$ for the incidental charge carriers of this polymeric resin.

The effect of SWCNT incorporation on the PVDF electrical conductivity is notable (Figure 6). For non-functionalized SWCNTs, a transition to a conductor state occurs at a concentration of 1-2 wt $\%$, according to a percolation mechanism. At $2 \mathrm{wt} \%$ SWCNTs, DC conductivity reaches a maximum value of $10^{-2} \mathrm{~S} / \mathrm{m}$, meaning an increase of almost nine orders of magnitude with respect to the insulting matrix. At higher filler concentrations, the conductivity levels off. The percolation behavior is also characterized by a significant shift of the critical frequency $\omega_{\mathrm{c}}$, from $30 \mathrm{~Hz}$ for $1 \mathrm{wt} \%$ concentration to around $10^{6} \mathrm{~Hz}$ for $3 \mathrm{wt} \%$. Such an effect is typical in disordered systems and usually follows a scaling power law dependence [55].

The incorporation of nanofillers also introduced other changes in the PVDF conductivity behavior. Figure 7 shows that $\sigma_{\mathrm{dc}}$ still follows an Arrhenius fashion in the temperature range where it is possible to clearly define it, but the activation energies decrease, compared to neat PVDF. In fact, the $E_{a}$ value changes with the addition of SWCNTs from 1.66 to $0.62 \mathrm{eV}$. On the other hand, the nitric acid treatment does not further modify $\mathrm{E}_{\mathrm{a}}$, while it increases to $\mathrm{E}_{\mathrm{a}}=1.25 \mathrm{eV}$ for SWCNT-PFO composites. 
In order to analyze the mechanisms involved in the conductivity, we calculated the exponent $\mathrm{s}$ of the Equation 3. Figure 8 depicts the $\sigma(\omega)$ curves of the composites that fulfil two conditions: i) the dielectric behavior is not relevant at the chosen temperature range $\left(-75^{\circ} \mathrm{C}\right.$ to $\left.150{ }^{\circ} \mathrm{C}\right)$, and ii) the dispersive $\sigma_{\omega} \omega^{\mathrm{s}}$ term is appreciable in the measured frequency range $\left(0.1 \mathrm{~Hz}\right.$ to $\left.10^{5} \mathrm{~Hz}\right)$. Both conditions are simultaneously fulfilled by the following materials: SWCNT-1/PVDF, SWCNT-PFO-4/PVDF and SWCNT-NIT5/PVDF. These composites are just in the middle way of the insulating-conductor transition with respect to the filler concentration. Here the values of the s parameter of Equation 3 indicate the nature of the conducting 3D clusters: s-values around 0.58 are related to anomalous diffusion in fractal clusters [56] and $\mathrm{s}=0.72$ indicates intercluster polarization [53]. In the composite with the untreated SWCNT nanofiller, the s-exponent ranges from 0.81 at $-75^{\circ} \mathrm{C}$ to 0.76 at $150^{\circ} \mathrm{C}$, indicating a behavior close to the intercluster polarization model. The decrease with the temperature does not appear in composites with SWCNT-NIT, whereas in SWCNT-PFO-4/PVDF the values change from 0.72 at $-25^{\circ} \mathrm{C}$ to 0.64 at $150{ }^{\circ} \mathrm{C}$, close to the diffusion in fractal cluster behavior. This trend is similar to that previously found by Ezquerra et al. [25] in SWCNT/PVDF composites, although in that report the s-value was calculated for nanofiller concentrations above the percolation concentration at a constant temperature. From our analysis, it can be additionally pointed that the increasing temperature activates an interparticle conduction mechanism in the composite, which is reminiscent of the above-commented "wave" effect in the dielectric constant $\varepsilon^{\prime}(\mathrm{T})$.

\section{Percolation threshold}

Figure 9 compares the conductivity at two different frequencies, $0.1 \mathrm{~Hz}$ and $10^{5}$ $\mathrm{Hz}$, and two temperatures, 25 and $150^{\circ} \mathrm{C}$, as a function of the nanofiller concentration. 
Figure 9a shows data at $25^{\circ} \mathrm{C}$ and $0.1 \mathrm{~Hz}$, in which we can observe that the influence of functionalized SWCNTs on conductivity follows a similar qualitative fashion to that generated by the incorporation of non-functionalized SWCNTs in the PVDF matrix. A percolation effect is observed in the three composites with the filler addition until reaching a maximum value of conductivity. However, in both the studied surface functionalization methods, the percolation threshold was higher than for non-functionalized SWCNTs, around $4 \mathrm{wt} \%$ for SWCNT-PFO and $6 \mathrm{wt} \%$ for SWCNT-NIT. The shift of the percolation threshold with SWCNT functionalization might be associated to several nanostructural phenomena: i) the functional groups may act as a coating layer on SWCNT surfaces [51]; ii) the electrical conductivity of individual SWCNTs may be locally hindered by surface functionalization due to the new chemical bonds; and iii) the SWCNT aspect ratio may decrease with chemical functionalization by oxidative breaking or aggregation induced by specific interactions between the functional groups [57, 58]. Apart from the percolation threshold shift, the outcomes indicate that the maximum attained conductivity was around $4 \times 10^{-3} \mathrm{~S} / \mathrm{m}$ regardless the surface modification undergone by SWCNTs. Therefore, once the electrical contact is stablished among the SWCNTs, the final conductivity is defined by the network characteristics but not by the intrinsic SWCNT properties. According to Ezquerra [25] this behavior can be extended to other carbon nanofillers above their respective percolation concentrations. The percolation critical concentration $\Phi_{\mathrm{c}}$ typically follows the trend $\sigma \sim\left(\Phi-\Phi_{\mathrm{c}}\right)^{\mathrm{t}}$, where $\mathrm{t}$ is a critical exponent, in the framework of the generalized percolation theory for CNTs in composite materials [59]. As above commented for the $\varepsilon^{\prime}(\Phi)$, the short number of specimen concentrations does not allow a calculation of an exact value for $\phi_{\mathrm{c}}$ in the present work.

Figures $9 \mathbf{b}$ and $9 \mathbf{c}$ respectively show the effect of frequency and temperature on the percolation threshold. The percolation at all the frequencies and temperatures occurs at 
nearly identical filler contents. However, the conductivity variation across the percolation threshold at high frequencies and temperatures is not as relevant as it is in Figure 9a. For instance, the conductivity at $10^{5} \mathrm{~Hz}$ and $25^{\circ} \mathrm{C}$ increased only by three orders of magnitude with respect to the neat polymer.

\section{CONCLUSIONS}

In the present work, SWCNT/ $\alpha-\mathrm{PVDF}$ composites are comprehensively characterized by dielectric relaxation spectroscopy (DRS). The influence of the SWCNT filler and the SWCNT chemical functionalization on the PVDF dielectric behavior and AC conductivity is described.

The $\alpha$ relaxation for the neat PVDF and all the composites appears in the temperature range of -40 to $-10^{\circ} \mathrm{C}$, and the fillers induce a small increase in the relaxation strength, which can be associated to a $10 \%$ decrease in crystallinity and a decrease in the relaxation time. The $\alpha_{c}$ relaxation appears for the neat PVDF and the composites between $10-80^{\circ} \mathrm{C}$. The SWCNT fillers produce a strong increase in the $\alpha_{c}$ dielectric strength, symmetry, broadness and the relaxation time.

The presence of SWCNT fillers greatly increases the dielectric constant $\varepsilon^{\prime}$ and the dielectric $\operatorname{loss}(\tan \delta)$ at filler concentrations above the electrical percolation threshold. Below the percolation, composites with functionalized SWCNTs demonstrate a moderate increase in $\varepsilon^{\prime}$ with negligible changes in tan $\delta$. Accordingly, composites below the percolation threshold may find application in capacitive systems while composites above the percolation threshold are known to be useful in electromagnetic shielding. The "wave" effect is observed in the $\varepsilon^{\prime}(\mathrm{T})$ plot of non-functionalized SWCNT composites. The electrical percolation threshold changes with the filler functionalization, and it is maintained in the whole measured temperature and frequency range. The maximum 
electrical conductivity above the percolation threshold is nearly identical $\left(\sim 10^{-2} \mathrm{~S} / \mathrm{m}\right)$ for all the SWCNT fillers, temperatures and frequencies.

\section{ACKNOWLEDGEMENTS}

Special thanks are directed to the Analysis Service of Instituto de Carboquímica, ICB-CSIC, in particular to I. Fernández. This work has been funded by MINECO and European Regional Development Fund (ENE 2016-79282-C5-1-R), and Government of Aragon and European Social Fund (DGA-T50 and DGA-ESF-T66 "Grupos Consolidados"). 


\section{FIGURE CAPTIONS}

Figure 1. XRD (left) and DSC (right) profiles for: (a) PVDF, (b) SWCNT-2/PVDF, (c) SWCNT-PFO-4.6/PVDF, and (d) SWCNT-NIT-7/PVDF.

Figure 2. 3 D representation showing the frequency/temperature dependence of PVDF: (a) real dielectric constant $\varepsilon^{\prime}$ and (b) loss dielectric constant $\varepsilon^{\prime \prime}$.

Figure 3. Isochronal curves of the complex dielectric constant, $\varepsilon^{\prime}$ and $\varepsilon^{\prime \prime}$, at $100 \mathrm{~Hz}(\mathrm{a}, \mathrm{b})$ and $10^{5} \mathrm{~Hz}(\mathrm{c}, \mathrm{d})$ respectively, for raw PVDF and composites below the percolation concentration.

Figure 4. Isothermal curves of $\varepsilon^{\prime \prime}$ spectra corresponding to the $\alpha$ relaxation from $-40{ }^{\circ} \mathrm{C}$ to $-10{ }^{\circ} \mathrm{C}$ (left) and $\alpha_{c}$ relaxation from $15^{\circ} \mathrm{C}$ to $75^{\circ} \mathrm{C}$ (right) of a) PVDF b) SWCNT-NIT5/PVDF and c) SWCNT-PFO-3/PVDF composites after subtracting the conductivity term. Solid lines correspond to the HN fit.

Figure 5. Temperature dependence of the Havriliak-Negami relaxation time for the processes in PVDF and in SWCNT/PDF composites: a) $\alpha$ relaxation and b) $\alpha_{c}$ relaxation. Continuous lines represent Arrhenius fittings.

Figure 6. Frequency dependence of the conductivity $\sigma(\omega)$ measured at room temperature and $150^{\circ} \mathrm{C}$ for virgin PVDF and their composites: a) SWCNT-X/PVDF, b) SWCNT-NITX/PVDF and c) SWCNT-POF-X/PVDF

Figure 7. Temperature dependence of $\sigma_{\mathrm{dc}}$ for the different SWCNT/PVDF composites. Continuous lines represent Arrhenius fittings.

Figure 8. Frequency dependence of the AC conductivity for various SWCNT/PVDF composites at different temperatures: $-75,-25,0,25,75,100$ and $150^{\circ} \mathrm{C}$.

Figure 9. Percolation effect in the SWCNT/PVDF composites at different temperatures and frequencies: a) $0.1 \mathrm{~Hz}$ and $25^{\circ} \mathrm{C}$, b) $10^{5} \mathrm{~Hz}$ and $25^{\circ} \mathrm{C}$ and c) $0.1 \mathrm{~Hz}$ and $150^{\circ} \mathrm{C}$. 
Table 1. SWCNT/PVDF composites studied in this work apart from the blank PVDF resin

\begin{tabular}{l|l|l}
\hline Composites & $\begin{array}{l}\text { Reinforcement } \\
\text { concentration }(\mathrm{X}=\mathrm{wt} \%)\end{array}$ & $\begin{array}{l}\text { Carbon nanotubes } \\
\text { functionalization }\end{array}$ \\
\hline SWCNT-X/PVDF & $1,2,3$ & Neat \\
\hline SWCNT-NIT-X/PVDF & $2,5,6,7,10$ & Nitric acid treatment \\
\hline SWCNT-PFO-X/PVDF & $1,3,4,4.6$ & $\begin{array}{l}\text { Heptadecafluoroctyl phenyl } \\
\text { groups }\end{array}$ \\
\hline
\end{tabular}

Table 2. DSC and XRD data for the virgin PVDF material and three selected SWCNT/PVDF composites: $\mathrm{T}_{\mathrm{m}}=$ melting temperature, $\Delta_{\mathrm{m}} \mathrm{H}=$ melting enthalpy, $\mathrm{T}_{\mathrm{c}}=$ crystallization temperature, $\Delta_{\mathrm{c}} \mathrm{H}=$ crystallization enthalpy, and $\mathrm{X}=$ crystallinity.

\begin{tabular}{lllllll}
\hline Material & $\begin{array}{l}\mathrm{T}_{\mathrm{m}} \\
{\left[{ }^{\circ} \mathrm{C}\right]}\end{array}$ & $\begin{array}{l}\Delta_{\mathrm{m}} \mathrm{H} \\
{\left[\mathrm{J} \mathrm{g}^{-1}\right]}\end{array}$ & $\begin{array}{l}\mathrm{T}_{\mathrm{c}} \\
{\left[{ }^{\circ} \mathrm{C}\right]}\end{array}$ & $\begin{array}{l}\Delta_{\mathrm{c}} \mathrm{H} \\
{\left[\mathrm{J} \mathrm{g} \mathrm{g}^{-1}\right]}\end{array}$ & $\begin{array}{l}\mathrm{X}_{\mathrm{DSC}} \\
{[\%]}\end{array}$ & $\begin{array}{l}\mathrm{X}_{\mathrm{XRD}} \\
{[\%]}\end{array}$ \\
\hline PVDF & 160.3 & -42.4 & 122.8 & 39.1 & 40.5 & 40.0 \\
SWCNT-2/PVDF & 162.3 & -39.6 & 137.6 & 34.0 & 37.8 & 33.7 \\
SWCNT-PFO-4.6/PVDF & 161.4 & -38.9 & 135.5 & 34.9 & 37.2 & 36.3 \\
SWCNT-NIT-7/PVDF & 161.2 & -36.7 & 135.4 & 33.2 & 35.1 & 29.6 \\
\hline
\end{tabular}

Table 3. Dielectric constant and loss tangent at two different frequencies, $1 \mathrm{~Hz}$ and $100000 \mathrm{~Hz}$, and temperatures, $25^{\circ} \mathrm{C}$ and $150^{\circ} \mathrm{c}$, corresponding two composites below and above the critical percolation concentration in each group.

\begin{tabular}{|c|c|c|c|c|c|c|c|c|}
\hline \multirow{2}{*}{$\begin{array}{l}\text { Material/Frequency- } \\
\text { Temperature }\end{array}$} & \multicolumn{2}{|c|}{$1 \mathrm{~Hz}-25^{\circ} \mathrm{C}$} & \multicolumn{2}{|c|}{$10^{5} \mathrm{~Hz}-25^{\circ} \mathrm{C}$} & \multicolumn{2}{|c|}{$1 \mathrm{~Hz}-150{ }^{\circ} \mathrm{C}$} & \multicolumn{2}{|c|}{$10^{5} \mathrm{~Hz}-150{ }^{\circ} \mathrm{C}$} \\
\hline & $\varepsilon^{\prime}$ & $\tan \delta$ & $\varepsilon^{\prime}$ & $\tan \delta$ & $\varepsilon^{\prime}$ & $\tan \delta$ & $\varepsilon^{\prime}$ & $\tan \delta$ \\
\hline PVDF & 12 & 0.07 & 9,4 & 0.04 & 29 & 4.1 & 11 & 0.06 \\
\hline SWCNT-1/PVDF & 124 & 1.16 & 21 & 0.16 & 192 & 7.6 & 24 & 0.2 \\
\hline SWCNT-2/PVDF & & & 105 & 4.11 & 5597 & 16687 & 85 & 8 \\
\hline SWCNT-PFO-3/PVDF & 22 & 0.07 & 16 & 0.06 & & 6.1 & 20 & 0,1 \\
\hline SWCNT-PFO-4.6/PVDF & 262 & 20370 & 50 & 1.39 & & 1901 & 68 & 2 \\
\hline SWCNT-NIT-5/PVDF & 14 & 0.07 & 13 & 0.04 & & 6.5 & 15 & 0.07 \\
\hline SWCNT-NIT-7/PVDF & 388 & 254 & 43 & 0.59 & & 212 & 58 & 0.63 \\
\hline
\end{tabular}


FIGURE 1. XRD (left) and DSC (right) profiles for: a) PVDF, b) SWCNT-2/PVDF, c) SWCNT-PFO-4.6/PVDF, and d) SWCNT-NIT-7/PVDF
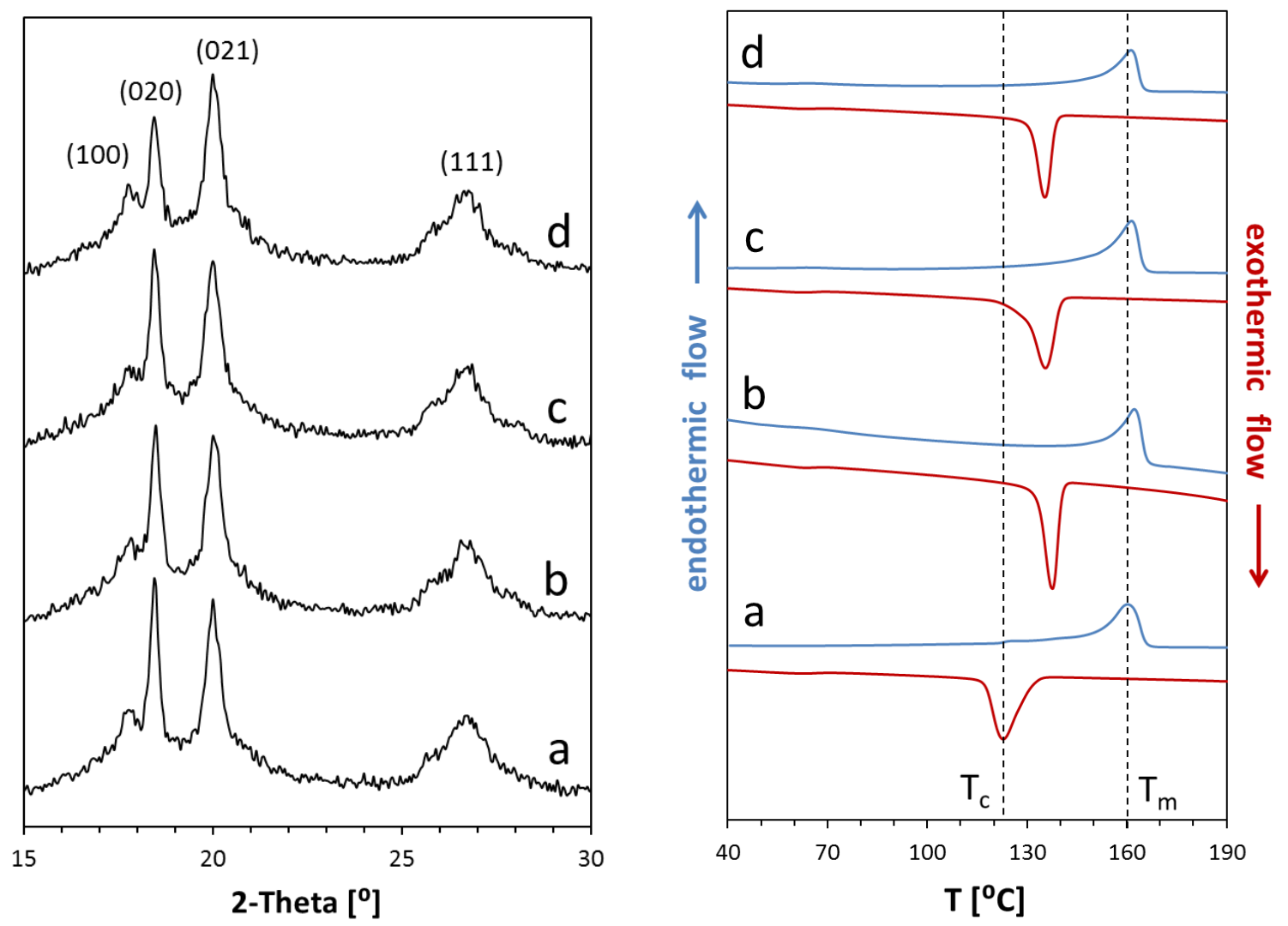
FIGURE 2. $3 \mathrm{D}$ representation showing the frequency/temperature dependence of PVDF: a) real dielectric constant $\varepsilon^{\prime}$. b) loss dielectric constant $\varepsilon^{\prime \prime}$.
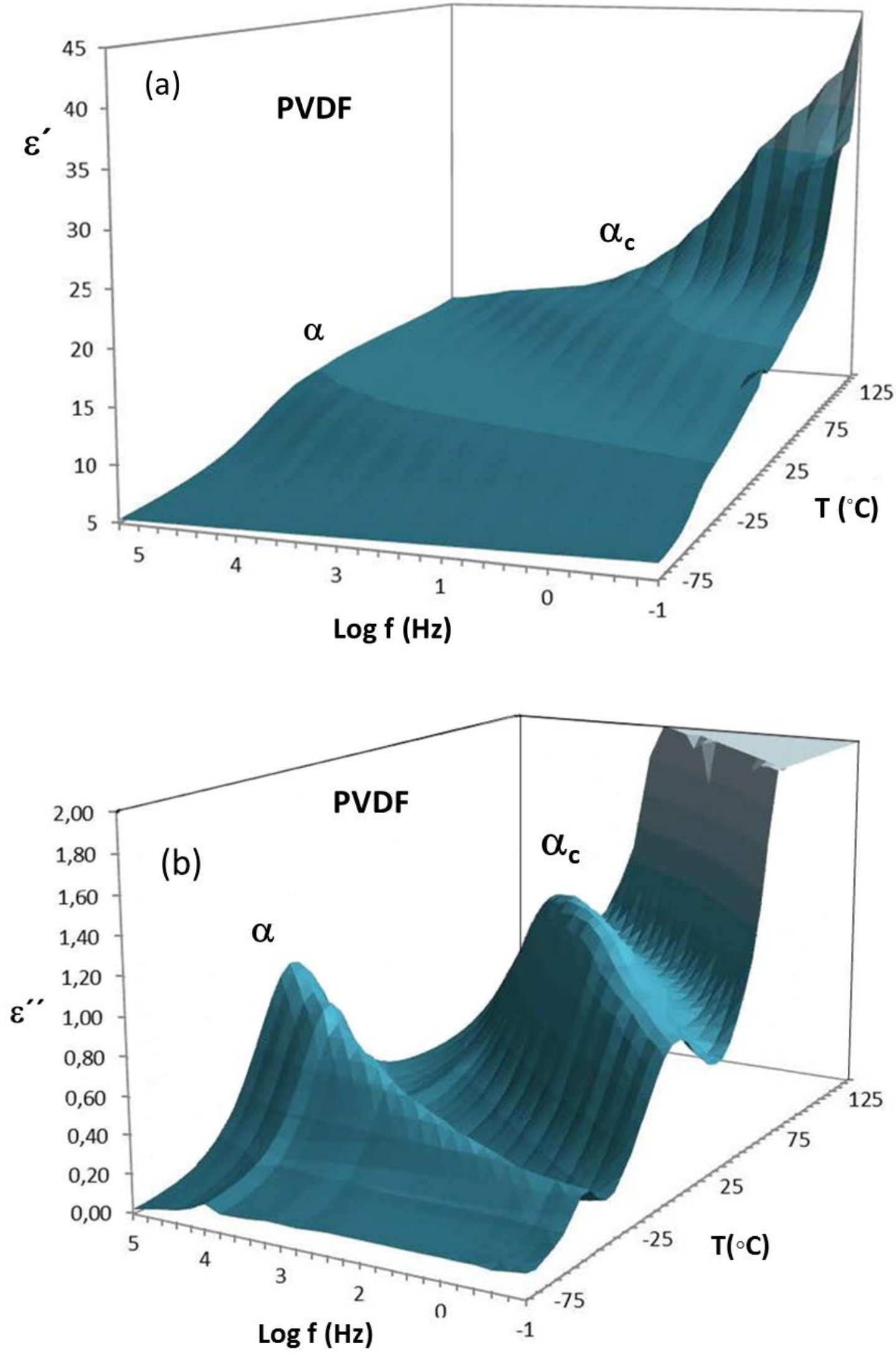
FIGURE 3. Isochronal curves of the complex dielectric constant, $\varepsilon^{\prime}$ and $\varepsilon^{\prime \prime}$, at $10 \mathrm{~Hz}(\mathrm{a}, \mathrm{b})$ and $10^{5} \mathrm{~Hz}(\mathrm{c}, \mathrm{d})$ respectively, for raw PVDF and composites below the percolation concentration.
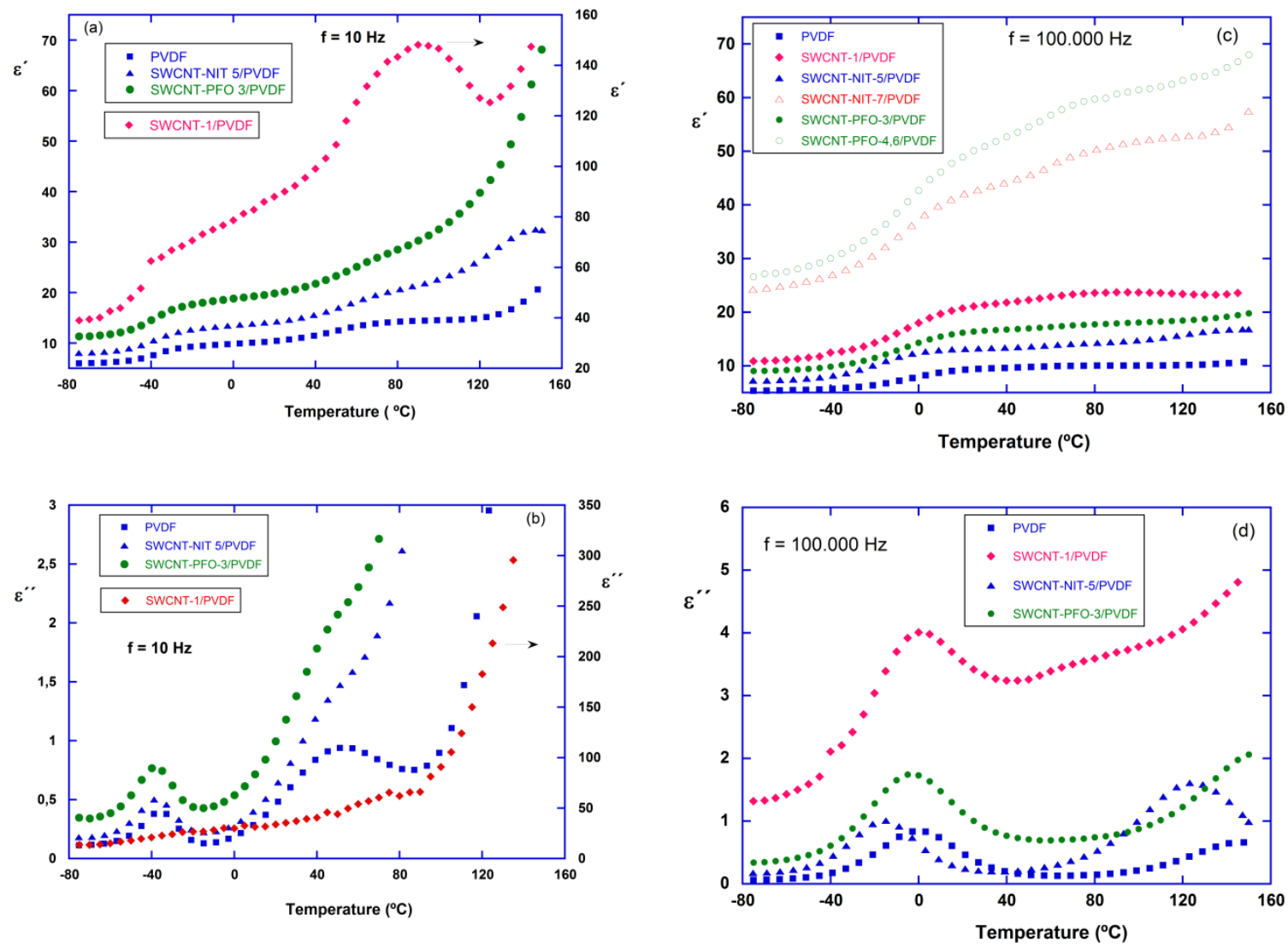
FIGURE 4. Isothermal curves of $\varepsilon^{\prime \prime}$ spectra corresponding to the $\alpha$ relaxation from -40 ${ }^{\circ} \mathrm{C}$ to $-10{ }^{\circ} \mathrm{C}$ (left) and $\alpha_{c}$ relaxation from $15{ }^{\circ} \mathrm{C}$ to $75^{\circ} \mathrm{C}$ (right) of a) PVDF b) SWCNTNIT-5/PVDF and c) SWCNT-PFO-3/PVDF composites after subtracting the conductivity term. Solid lines correspond to the HN fit.
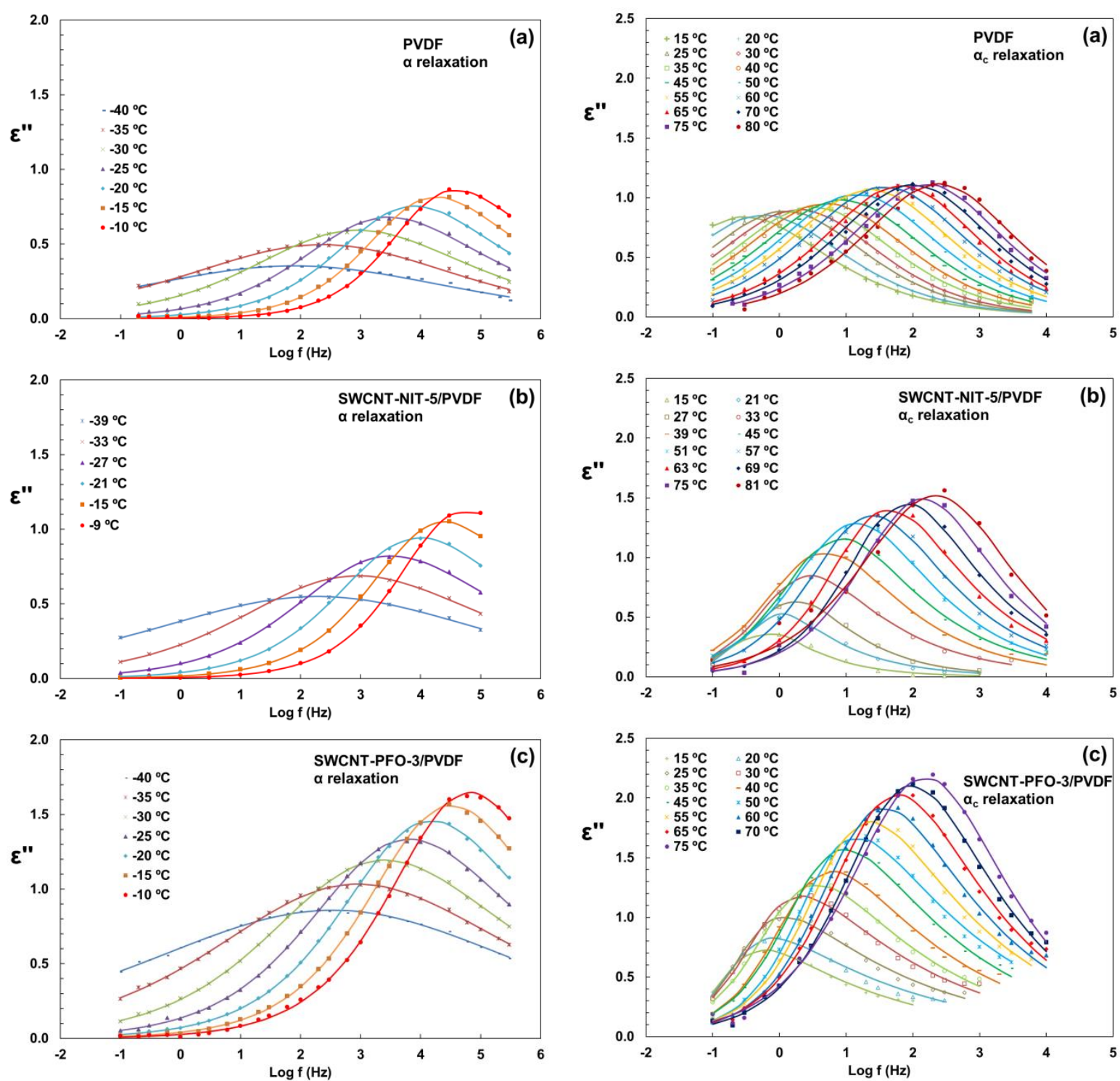
FIGURE 5. . Temperature dependence of the Havriliak-Negami relaxation time for the processes in PVDF and in SWCNT/PDF composites: a) $\alpha$ relaxation and b) $\alpha_{c}$ relaxation. Continuous lines represent Arrhenius fittings.
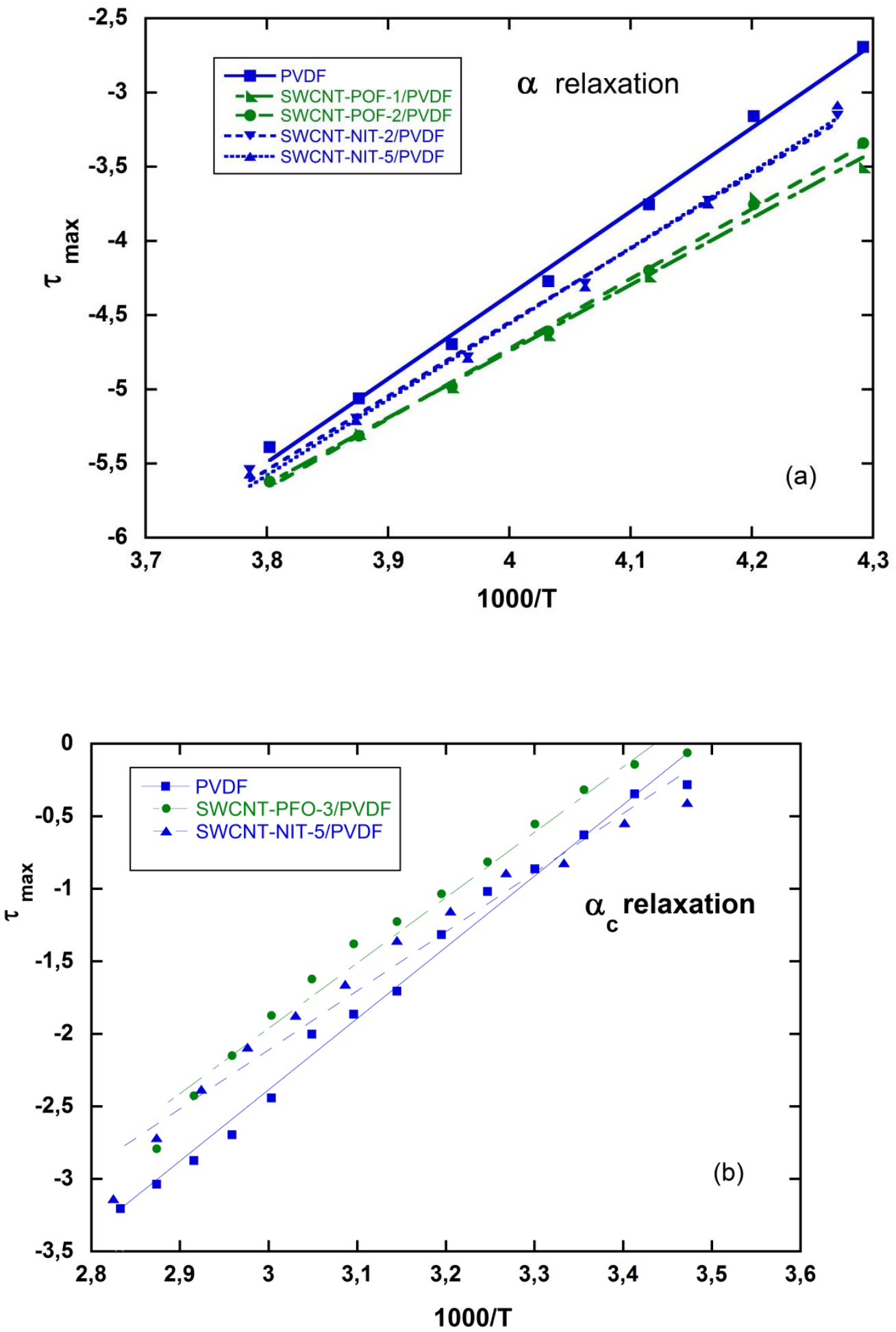
FIGURE 6. Frequency dependence of the conductivity $\sigma(\omega)$ measured at room temperature and $150{ }^{\circ} \mathrm{C}$ for virgin PVDF and their composites: a) SWCNT-X/PVDF, b) SWCNT-NIT-X/PVDF and c) SWCNT-PFO-X/PVDF
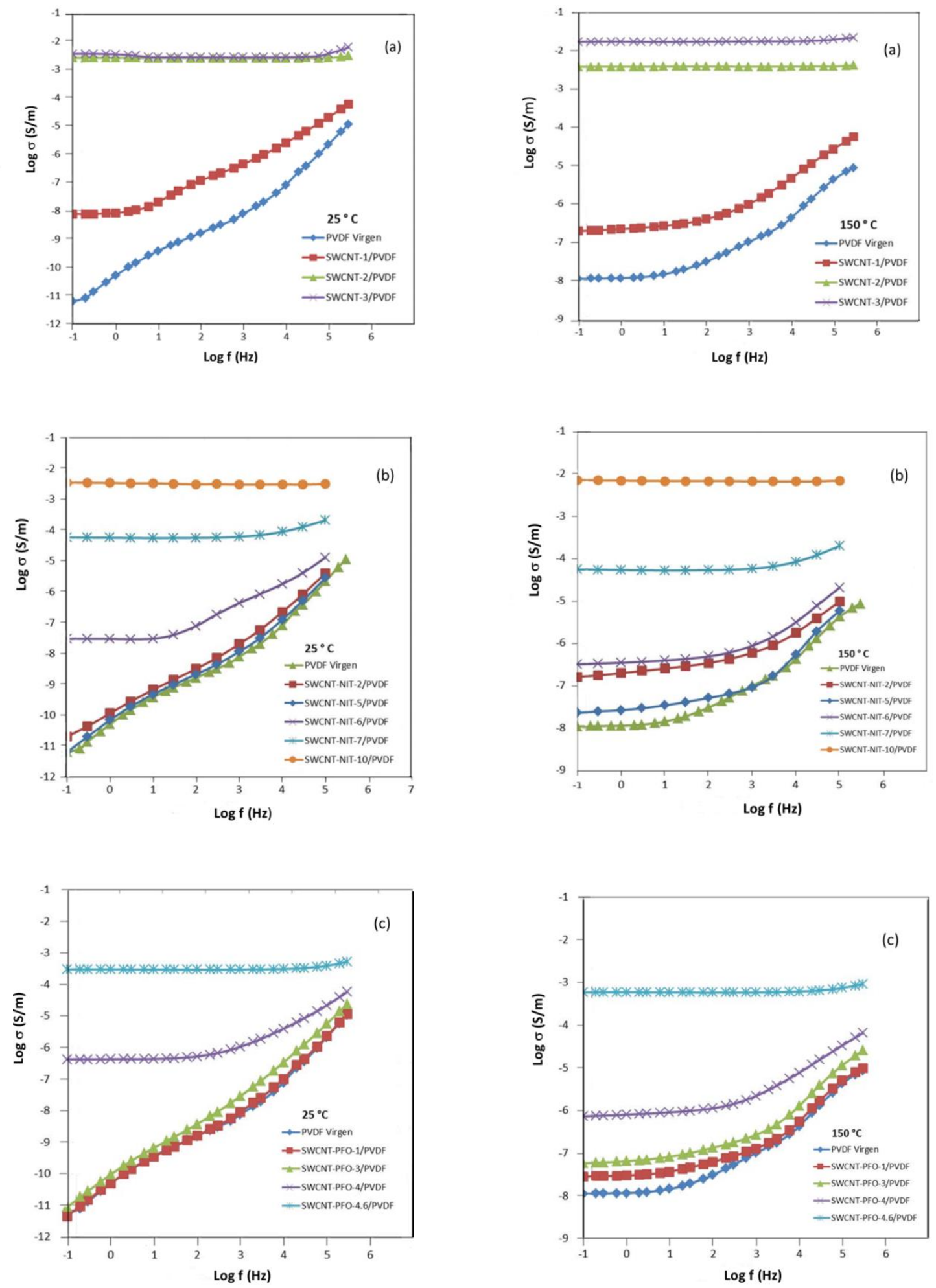
Figure 7. Temperature dependence of $\sigma_{\mathrm{dc}}$ for the different SWCNT/PVDF composites. Continuous lines represent Arrhenius fittings.

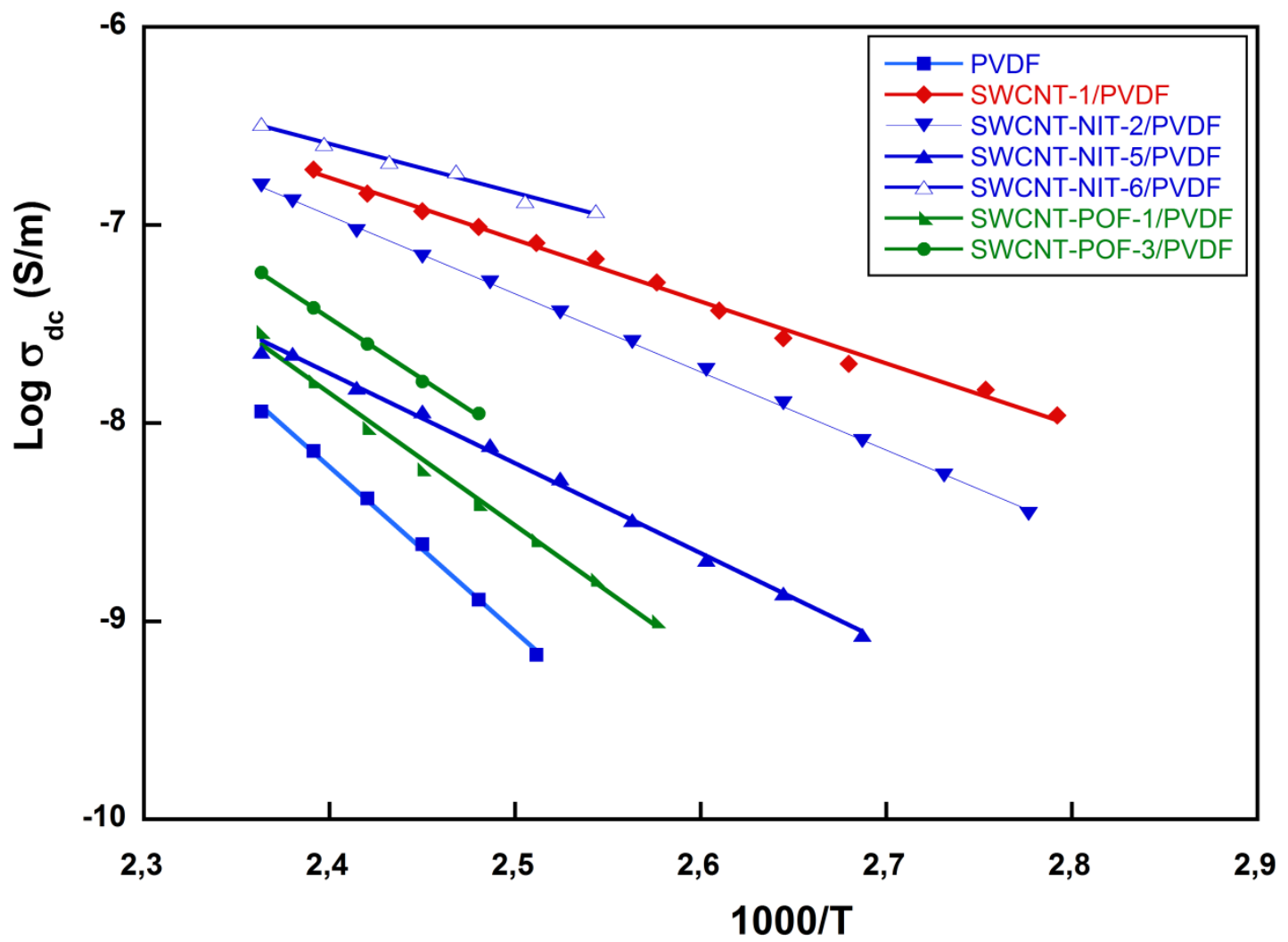


FIGURE 8. Frequency dependence of the AC conductivity for various SWCNT/PVDF composites at different temperatures: -75, -25, 0, 25, 75, 100 and $150^{\circ} \mathrm{C}$.

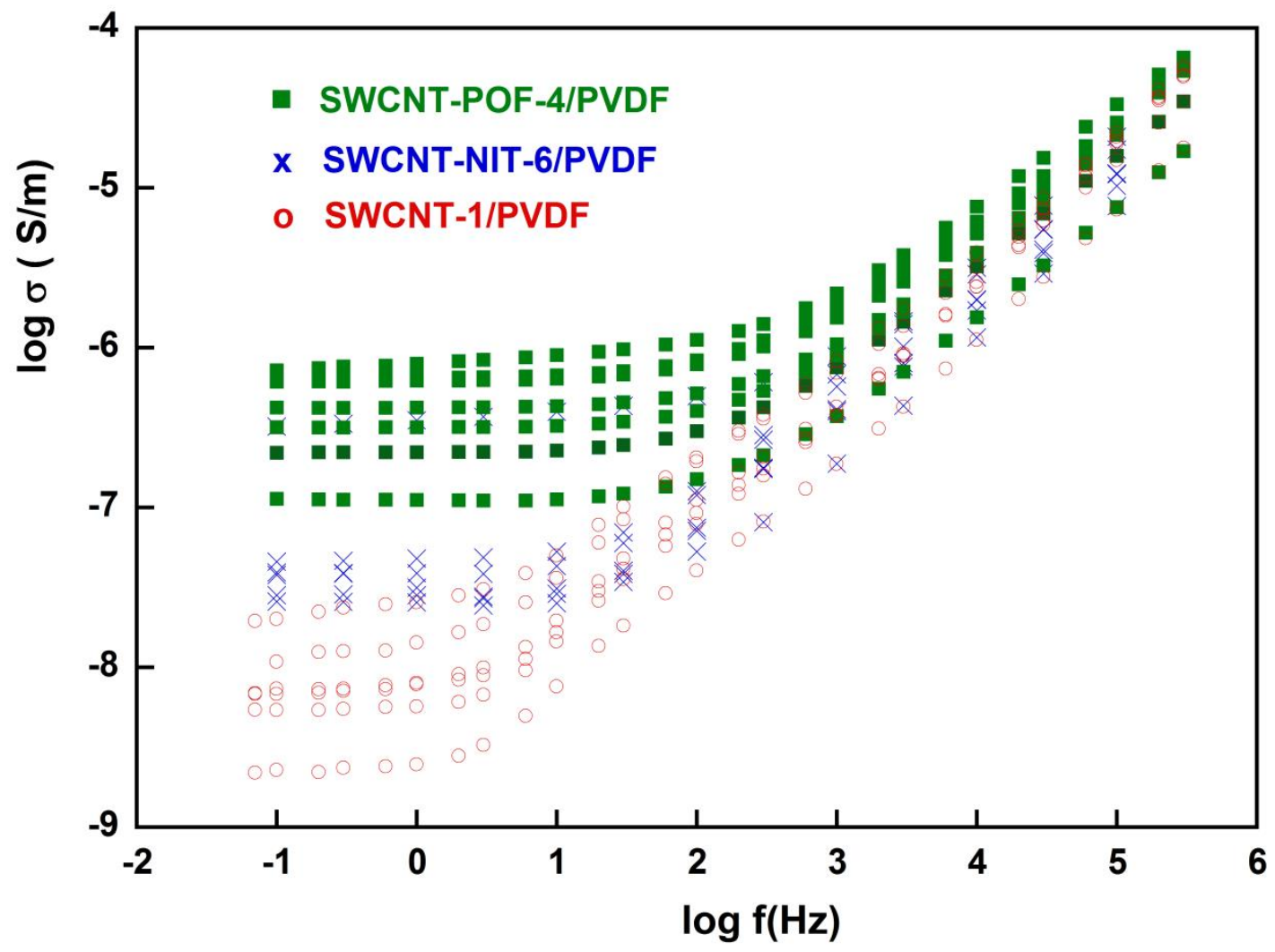


FIGURE 9. Percolation effect in the SWCNT/PVDF composites at different temperatures and frequencies: a) $0.1 \mathrm{~Hz}$ and $25^{\circ} \mathrm{C}$, b) $10^{5} \mathrm{~Hz}$ and $25^{\circ} \mathrm{C}$ and c) $0.1 \mathrm{~Hz}$ and $150^{\circ} \mathrm{C}$.
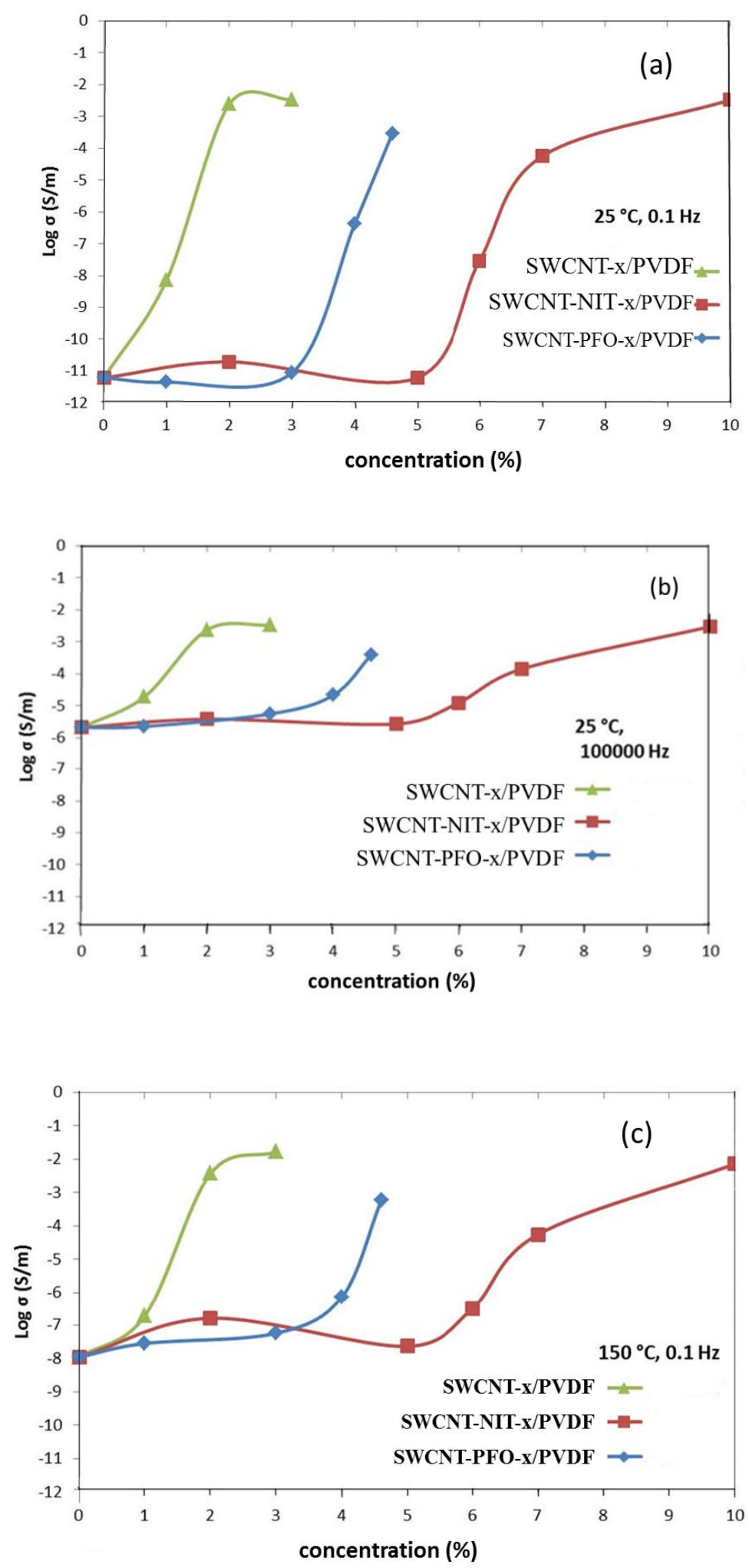


\section{REFERENCES}

[1] X. Chen, X. Han, Q.D. Shen, PVDF-Based Ferroelectric Polymers in Modern Flexible Electronics, Adv Electron Mater 3(5) (2017).

[2] H.M. Yang, Y.K. Kwon, S.B. Lee, S. Kim, K. Hong, K.H. Lee, Physically CrossLinked Homopolymer Ion Gels for High Performance Electrolyte-Gated Transistors, Acs Applied Materials \& Interfaces 9(10) (2017) 8813-8818.

[3] E. Fontananova, V. Grosso, S.A. Aljlil, M.A. Bahattab, D. Vuono, G. Di Profio, E. Drioli, Polyvinylidenefluoride/carbon nanotubes mixed matrix membranes with tailored properties, Aip Conf Proc 1736 (2016).

[4] Y.L. Yu, H. Chen, Y. Liu, V.S.J. Craig, L.H. Li, Y. Chen, A. Tricoli, Porous carbon nanotube/polyvinylidene fluoride composite material:

Superhydrophobicity/superoleophilicity and tunability of electrical conductivity, Polymer 55(22) (2014) 5616-5622.

[5] U. Staudinger, P. Thoma, F. Luttich, A. Janke, O. Kobsch, O.D. Gordan, P.

Potschke, B. Voit, D.R.T. Zahn, Properties of thin layers of electrically conductive polymer/MWCNT composites prepared by spray coating, Composites Science and Technology 138 (2017) 134-143.

[6] C. Yang, Y.H. Lin, C.W. Nan, Modified carbon nanotube composites with high dielectric constant, low dielectric loss and large energy density, Carbon 47(4) (2009) 1096-1101.

[7] M.H. Yang, H. Zhao, D.L. He, J.B. Bai, Constructing a continuous amorphous carbon interlayer to enhance dielectric performance of carbon nanotubes/polyvinylidene fluoride nanocomposites, Carbon 116 (2017) 94-102. [8] A.K. Batra, M.E. Edwards, P. Guggilla, M.D. Aggarwal, R.B. Lal, Pyroelectric Properties of PVDF:MWCNT Nanocomposite Film for Uncooled Infrared Detectors and Medical Applications, Integr Ferroelectr 158(1) (2014) 98-107.

[9] H. Wang, K. Zheng, X. Zhang, T.X. Du, C. Xiao, X. Ding, C. Bao, L. Chen, X.Y. Tian, Segregated poly(vinylidene fluoride)/MWCNTs composites for highperformance electromagnetic interference shielding, Compos Part a-Appl S 90 (2016) 606-613.

[10] J. Bernal-Martinez, A. Sesena-Rubfiaro, R. Godinez-Fernandez, A. AguilarElguezabal, Electrodes made of multi-wall carbon nanotubes on PVDF-filters have low electrical resistance and are able to record electrocardiograms in humans, Microelectron Eng 166 (2016) 10-14.

[11] A. Ferrreira, J.G. Rocha, A. Anson-Casaos, M.T. Martinez, F. Vaz, S. LancerosMendez, Electromechanical performance of poly(vinylidene fluoride)/carbon nanotube composites for strain sensor applications, Sensor Actuat a-Phys 178 (2012) 10-16.

[12] S. Mishra, K.T. Kumaran, R. Sivakumaran, S.P. Pandian, S. Kundu, Synthesis of PVDF/CNT and their functionalized composites for studying their electrical properties to analyze their applicability in actuation \& sensing, Colloid Surface $A$ 509 (2016) 684-696.

[13] K. Ravikumar, G.P. Kar, S. Bose, B. Basu, Synergistic effect of polymorphism, substrate conductivity and electric field stimulation towards enhancing muscle cell growth in vitro, Rsc Adv 6(13) (2016) 10837-10845.

[14] K. Ke, P. Potschke, N. Wiegand, B. Krause, B. Voit, Tuning the Network Structure in Poly(vinylidene fluoride)/Carbon Nanotube Nanocomposites Using Carbon Black: Toward Improvements of Conductivity and Piezoresistive Sensitivity, Acs Applied Materials \& Interfaces 8(22) (2016) 14190-14199. 
[15] M.F.L. De Volder, S.H. Tawfick, R.H. Baughman, A.J. Hart, Carbon Nanotubes: Present and Future Commercial Applications, Science 339(6119) (2013) 535-539.

[16] S. Begum, A. Kausar, H. Ullah, M. Siddiq, Potential of Polyvinylidene Fluoride/Carbon Nanotube Composite in Energy, Electronics, and Membrane Technology: An Overview, Polym-Plast Technol 55(18) (2016) 1949-1970. [17] M. Arjmand, U. Sundararaj, Impact of BaTiO3 as Insulative Ferroelectric Barrier on the Broadband Dielectric Properties of MWCNT/PVDF Nanocomposites, Polym Composite 37(1) (2016) 299-304.

[18] A.B. da Silva, M. Arjmand, U. Sundararaj, R.E.S. Bretas, Novel composites of Copper nanowire/PVDF with superior dielectric properties, Polymer 55(1) (2014) 226-234.

[19] M. Arjmand, S. Sadeghi, M. Khajehpour, U. Sundararaj, Carbon Nanotube/Graphene Nanoribbon/Polyvinylidene Fluoride Hybrid Nanocomposites: Rheological and Dielectric Properties, Journal of Physical Chemistry C 121(1) (2017) 169-181.

[20] G.S. Kumar, D. Vishnupriya, K.S. Chary, T.U. Patro, High dielectric permittivity and improved mechanical and thermal properties of poly(vinylidene fluoride) composites with low carbon nanotube content: effect of composite processing on phase behavior and dielectric properties (vol 27, 385702, 2016), Nanotechnology 27(43) (2016).

[21] A. Ameli, M. Arjmand, P. Potschke, B. Krause, U. Sundararaj, Effects of synthesis catalyst and temperature on broadband dielectric properties of nitrogendoped carbon nanotube/polyvinylidene fluoride nanocomposites, Carbon 106 (2016) 260-278.

[22] C. Seoul, Y.T. Kim, C.K. Baek, Electrospinning of poly(vinylidene fluoride)/dimethylformamide solutions with carbon nanotubes, J Polym Sci Pol Phys 41(13) (2003) 1572-1577.

[23] F.J. Owens, J.R.P. Jayakody, S.G. Greenbaum, Characterization of single walled carbon nanotube: Polyvinylene difluoride composites, Composites Science and Technology 66(10) (2006) 1280-1284.

[24] A. Anson-Casaos, J.M. Gonzalez-Dominguez, A.M. Diez-Pascual, M.A. GomezFatou, M.T. Martinez, Choosing the Chemical Route for Carbon Nanotube Integration in Poly(vinylidene fluoride), Journal of Physical Chemistry C 116(30) (2012) 16217-16225.

[25] T.A. Ezquerra, J.C. Canalda, A. Sanz, A. Linares, On the electrical conductivity of PVDF composites with different carbon-based nanoadditives, Colloid Polym Sci 292(8) (2014) 1989-1998.

[26] V. Sencadas, S. Lanceros-Mendez, J.F. Mano, Characterization of poled and non-poled beta-PVDF films using thermal analysis techniques, Thermochim Acta 424(1-2) (2004) 201-207.

[27] S. Havriliak, S. Negami, A Complex Plane Analysis of Alpha-Dispersions in Some Polymer Systems, J Polym Sci Pol Sym (14PC) (1966) 99-+.

[28] K.L. Ngai, A. Schonhals, E. Schlosser, An Explanation of Anomalous DielectricRelaxation Properties of Poly(Propylene Glycol), Macromolecules 25(19) (1992) 4915-4919.

[29] A.K. Jonscher, Universal Dielectric Response, Nature 267(5613) (1977) 673-679. [30] A. Almasri, Z. Ounaies, Y.S. Kim, J. Grunlan, Characterization of solutionprocessed double-walled carbon nanotube/poly(vinylidene fluoride) nanocomposites, Macromol Mater Eng 293(2) (2008) 123-131. 
[31] I.H. Kim, D.H. Baik, Y.G. Jeong, Structures, electrical, and dielectric properties of PVDF-based nanocomposite films reinforced with neat multi-walled carbon nanotube, Macromol Res 20(9) (2012) 920-927.

[32] M. Sharma, G. Madras, S. Bose, Size dependent structural relaxations and dielectric properties induced by surface functionalized MWNTs in poly(vinylidene fluoride)/poly(methyl methacrylate) blends, Phys Chem Chem Phys 16(6) (2014) 2693-2704.

[33] S.K. Rath, S. Dubey, G.S. Kumar, S. Kumar, A.K. Patra, J. Bahadur, A.K. Singh, G. Harikrishnan, T.U. Patro, Multi-walled CNT-induced phase behaviour of poly(vinylidene fluoride) and its electro-mechanical properties, Journal of Materials Science 49(1) (2014) 103-113.

[34] Y. Ahn, J.Y. Lim, S.M. Hong, J. Lee, J. Ha, H.J. Choi, Y. Seo, Enhanced Piezoelectric Properties of Electrospun Poly(vinylidene fluoride)/Multiwalled Carbon Nanotube Composites Due to High beta-Phase Formation in Poly(vinylidene fluoride), Journal of Physical Chemistry C 117(22) (2013) 11791-11799.

[35] E.K. Kabir, M.; Nasrin, L.; Raihan, M.J.; Rahman, M. , Pure $\beta$-phase formation in polyvivylidene fluoride (PVDF)-carbon nanotube composites. , J. Phys. D: Appl. Phys. 50 (2017).

[36] M. Sharma, K. Sharma, S. Bose, Segmental Relaxations and CrystallizationInduced Phase Separation in PVDF/PMMA Blends in the Presence of SurfaceFunctionalized Multiwall Carbon Nanotubes, J Phys Chem B 117(28) (2013) 85898602.

[37] V. Sencadas, S. Lanceros-Mendez, R.S.I. Serra, A.A. Balado, J.L.G. Ribelles, Relaxation dynamics of poly(vinylidene fluoride) studied by dynamical mechanical measurements and dielectric spectroscopy, Eur Phys J E 35(5) (2012).

[38] Y. Ishida, M. Watanabe, K. Yamafuji, Dielectric Behavior of Polyvinylidene Fluoride, Kolloid Z Z Polym 200(1) (1964) 48-\&.

[39] A. Hassen, T. Hanafy, S. El-Sayed, A. Himanshu, Dielectric relaxation and alternating current conductivity of polyvinylidene fluoride doped with lanthanum chloride, J Appl Phys 110(11) (2011).

[40] J. Kaszynska, B. Hilczer, P. Biskupski, Segmental dynamics in poly(vinylidene fluoride) studied by dielectric, mechanical and nuclear magnetic resonance spectroscopies, Polym Bull 68(4) (2012) 1121-1134.

[41] H. Kakutani, Dielectric Absorption in Oriented Poly(Vinylidene Fluoride), J Polym Sci A2 8(7) (1970) 1177-\&.

[42] T.U. Patro, M.V. Mhalgi, D.V. Khakhar, A. Misra, Studies on poly(vinylidene fluoride)-clay nanocomposites: Effect of different clay modifiers, Polymer 49(16) (2008) 3486-3499.

[43] N. Alves, C.X. Cardoso, A.E. Job, J.A. Giacometti, Effects of thermal treatment on phase transitions and on the mechanical relaxation in poly(Vinylidene fluoride), 11th International Symposium on Electrets (Ise 11) (2002) 263-266.

[44] J.K. Yuan, S.H. Yao, Z.M. Dang, A. Sylvestre, M. Genestoux, J.B. Bai, Giant Dielectric Permittivity Nanocomposites: Realizing True Potential of Pristine Carbon Nanotubes in Polyvinylidene Fluoride Matrix through an Enhanced Interfacial Interaction, Journal of Physical Chemistry C 115(13) (2011) 5515-5521.

[45] R. Gregorio, E.M. Ueno, Effect of crystalline phase, orientation and temperature on the dielectric properties of poly (vinylidene fluoride) (PVDF), Journal of Materials Science 34(18) (1999) 4489-4500.

[46] L. Yang, J.H. Qiu, K.J. Zhu, H.L. Ji, Effect of rolling on structure and ferroelectric properties of poly(vinylidenefluoride) with presence of multiwalled carbon nanotubes, Mater Res Innov 18 (2014) 564-569. 
[47] J. Mijovic, J.W. Sy, T.K. Kwei, Reorientational dynamics of dipoles in poly (vinylidene fluoride) poly(methyl methacrylate) (PVDF/PMMA) blends by dielectric spectroscopy, Macromolecules 30(10) (1997) 3042-3050.

[48] A. Linares, A. Nogales, D.R. Rueda, T.A. Ezquerra, Molecular dynamics in PVDF/PVA blends as revealed by dielectric loss spectroscopy, J Polym Sci Pol Phys 45(13) (2007) 1653-1661.

[49] V.J. Mcbrierty, D.C. Douglass, T.A. Weber, Nuclear Magnetic-Relaxation and Molecular-Motion in Poly(Vinylidine Fluoride), J Polym Sci Pol Phys 14(7) (1976) 1271-1286.

[50] Z.M. Dang, S.H. Yao, H.P. Xu, Effect of tensile strain on morphology and dielectric property in nanotube/polymer nanocomposites, Appl Phys Lett 90(1) (2007).

[51] Z.M. Dang, L. Wang, Y. Yin, Q. Zhang, Q.Q. Lei, Giant dielectric permittivities in functionalized carbon-nanotube/electroactive-polymer nanocomposites, Adv Mater 19(6) (2007) 852-+.

[52] Q. Li, Q.Z. Xue, L.Z. Hao, X.L. Gao, Q.B. Zheng, Large dielectric constant of the chemically functionalized carbon nanotube/polymer composites, Composites Science and Technology 68(10-11) (2008) 2290-2296.

[53] D.J. Bergman, Y. Imry, Critical Behavior of Complex Dielectric-Constant near Percolation Threshold of a Heterogeneous Material, Phys Rev Lett 39(19) (1977) 1222-1225.

[54] Q. Li, Q.Z. Xue, X.L. Gao, Q.B. Zheng, Temperature dependence of the electrical properties of the carbon nanotube/polymer composites, Express Polym Lett 3(12) (2009) 769-777.

[55] J.C. Dyre, T.B. Schroder, Universality of ac conduction in disordered solids, Rev Mod Phys 72(3) (2000) 873-892.

[56] Y. Gefen, A. Aharony, S. Alexander, Anomalous Diffusion on Percolating Clusters, Phys Rev Lett 50(1) (1983) 77-80.

[57] S.H. Yao, Z.M. Dang, M.J. Jiang, H.P. Xu, J.B. Bai, Influence of aspect ratio of carbon nanotube on percolation threshold in ferroelectric polymer nanocomposite, Appl Phys Lett 91(21) (2007).

[58] F.H. Su, M.H. Miao, Effect of MWCNT dimension on the electrical percolation and mechanical properties of poly(vinylidenefluoride-hexafluoropropylene) based nanocomposites, Synthetic Met 191 (2014) 99-103.

[59] W. Bauhofer, J.Z. Kovacs, A review and analysis of electrical percolation in carbon nanotube polymer composites, Composites Science and Technology 69(10) (2009) 1486-1498. 